\title{
CONCERNING END POINTS OF CONTINUOUS CURVES AND OTHER CONTINUA*
}

\author{
BY \\ HARRY MERRILL GEHMAN†
}

\section{INTRODUCTION}

Several authors have given definitions of an end point of a continuum, making use of properties which are possessed by an end point of a straight line interval, but which are not possessed by any interior point of the interval. We shall show in the present paper that the properties used in these so-called "definitions" are not logically equivalent, and shall determine the logical relations which do exist among these properties under various conditions. In Part 2, we shall show the equivalence of a number of these properties in the case of a continuous curve. In Part 3 is shown the equivalence with the first set, of two additional properties in the case of a continuous curve of a special type. In Parts 4 and 5, we determine the logical relations existing among certain of these properties in the case of a bounded continuum. Finally, in Part 6, some theorems are proved concerning points having one or more of the given properties.

In this paper we shall consider only plane point sets, although in a number of cases it is obvious that our results are true in space of any number of dimensions.

In regard to the use of the word "end point," we intend hereafter to use this word only in the sense of a point of a continuous curve satisfying Wilder's definition or one of the other definitions equivalent to it, in other words, a point having any one of the properties 1-7 given in Part 2. We shall not use the word "end point" in referring to a point of a continuum which is not a continuous curve. The examples of Part 4 show that a point of a continuum may have certain of the given properties and yet be so placed with respect to that continuum as hardly to deserve the name of "end point."

\section{CONCERNING PROPERTIES 1-7 FOR A CONTINUOUS CURVE}

The object of this section is to prove the equivalence of the following

* Presented to the Society, April 2 and September 9, 1926; received by the editors August 10, 1926.

† National Research Fellow in Mathematics. 
properties of a point of a continuous curve.* In each case, $P$ denotes a point of a continuous curve $M$.

Property 1. If $P P^{\prime}$ is any arc in $M$ whose end points are $P$ and any other point $P^{\prime}$ of $M$, then the set $M-\left(P P^{\prime}-P\right)$ contains no connected subset consisting of more than one point which contains $P$.

Property 2. If $P P^{\prime}$ is any arc in $M$ whose end points are $P$ and any other point $P^{\prime}$ of $M$, then $P$ is not a limit point of any connected subset of $M-P P^{\prime}$.

Property 3. If $N$ is any subcontinuum of $M$ containing $P$, then the set $M-(N-P)$ contains no connected subset consisting of more than one point which contains $P$.

Property 4. $P$ is not a cut point $f$ of any subcontinuum of $M$.

Property 5. $P$ is not contained in any subcontinuum of $M$ which is irreducible $\ddagger$ between two other points of $M$.

Property 6. If $N$ is any subcontinuum of $M$ containing $P$, then $P$ is not a limit point of any connected subset of $M-N$.

Property 7. Given any positive number $\epsilon$, there exists a domain containing $P$ of diameter less than $\epsilon$, whose boundary has just one point in common with $M$.

Property 1 is due to R.L. Wilder. $\S$ Properties 2, 3, and 6 are modifications of property 1. Property 4 was suggested by Professor R. L. Moore. Property 5 is due to Yoneyama.\| Property 7 is due to Menger. I

G. T. Whyburn** has proved that in the case of a continuous curve, property 1 is equivalent to the following property: No arc in $M$ contains $P$ as an interior point. In the case of a continuum which is not a continuous

* For a number of equivalent definitions of a continuous curve, see R. L. Moore, Report on continuous curves from the viewpoint of analysis situs, Bulletin of the American Mathematical Society, vol. 29 (1923), pp. 289-302. In the present paper we shall make one change in the definitions given in Report, i.e., we shall define a continuum as a closed and connected point set containing more than one point.

† If $M$ is a connected point set, and $P$ is a point of $M$, then if $M-P$ is not connected, $P$ is said to be a cut point of $M$; if $M-P$ is connected $P$ is said to be a non-cut point of $M$.

¥ A point set $K$ is said to be an irreducible continuum between two points $A$ and $B$, if $K$ is a continuum and contains $A$ and $B$, but contains no proper subset which is a continuum and contains $A$ and $B$.

8 R. L. Wilder, Concerning continuous curves, Fundamenta Mathematicae, vol. 7 (1925), pp. 340377. See especially p. 358.

|| K. Yoneyama, Theory of continuous set (sic) of points, Tôhoku Mathematical Journal, vol. 13 (1918), p. 130.

IK. Menger, Grundzüge einer Theorie der Kurven, Mathematische Annalen, vol. 95 (1925), pp. 277-306.

**G. T. Whyburn, Concerning continua in the plane, these Transactions, vol. 29 (1927), pp. 369 400. See Theorem 12, p. 385. 
curve, Whyburn uses property 6 as a definition of an end point of the continuum.

W. L. Ayres* has proved that in the case of a continuous curve, property 1 is equivalent to the following property: $P$ is a non-cut point of $M$ which belongs to no simple closed curve in $M$.

Theorem 1. If a point $P$ of a continuous curve $M$ has any one of the properties 1-7, it has all the others.

In Part 4 of this paper, we shall show that if $M$ is any bounded continuum, and $P$ has property 7 , it has property 6 ; if $P$ has property 6 , it has property 5 ; if $P$ has property 5 , it has property 4 .

If $P$ has property 4 , it has property 2 . For if it fails to have property 2 , then $M$ contains an arc $P P^{\prime}$, such that $P$ is a limit point of a connected subset $X$ of $M-P P^{\prime}$. Let $K$ denote the maximal connected subset of $M-P P^{\prime}$ containing $X$. The point $P$ can be joined to any point $P^{\prime \prime}$ of $K$ by an arct lying in $K$ except for the point $P$, and therefore having only $P$ in common with the $\operatorname{arc} P P^{\prime}$. The sum of the $\operatorname{arcs} P P^{\prime}$ and $P P^{\prime \prime}$ is an $\operatorname{arc} P^{\prime} P^{\prime \prime}$ of which $P$ is an interior point, and therefore a cut point. But this is contrary to the assumption that $P$ has property 4 .

If $P$ has property 2, it has property 1 . For if it fails to have property 1, then there is some arc $P P^{\prime}$ in $M$, such that $M-\left(P P^{\prime}-P\right)$ contains a connected subset $X$ containing $P$ and such that $X-P$ is not vacuous. Let $K$ be the maximal connected subset of $M-\left(P P^{\prime}-P\right)$ containing $P$. Since $K$ contains $X$, the set $K-P$ is not vacuous. If $Q$ is any point of $K-P$, then $K$ contains the maximal connected subset of $M-P P^{\prime}$ containing $Q$. Let us denote this set by $D$. Since the point $P$ has property $2, P$ is not a limit point of $D$, and therefore $K$ contains other points besides $P$ and points of $D$. The set $D$ is closed, save for limit points on $P P^{\prime}$, and since the point $P$ is not a limit point of $D$, no point of $K-D$ is a limit point of $D$. Also, no point of $D$ can be a limit point of any set of points of $M$ not in $D \ddagger$, and therefore no point of $D$ is a limit point of $K-D$. Therefore $K$ is disconnected, which is contrary to our supposition concerning $K$.

If in the argument in the preceding paragraph, we replace the $\operatorname{arc} P P^{\prime}$ by a subcontinuum $N$ of $M$ containing $P$, we can prove that if $P$ has property 6 , it has property 3 . Obviously if $P$ has property 3 , it has property 6 , and

*W. L. Ayres, Concerning continuous curves and correspondences, Annals of Mathematics, (2), vol. 28 (1927), pp. 396-418. See Theorem 3, p. 399.

† R. L. Wilder, loc. cit., Theorem 1, p. 342.

$\ddagger$ R. L. Wilder, loc. cit. See the proof of Theorem 9, p. 360. 
therefore properties 3 and 6 are equivalent. To complete the proof of Theorem 1, we need only show that if $P$ has property 1 , it has property 7 .

If $P$ has property 1 , and $P P^{\prime}$ is any $\operatorname{arc}$ in $M$, then $P$ is a limit point of a set of points $L$ of $P P^{\prime}-\left(P+P^{\prime}\right)$, such that if $X$ is any point of $L$, then $P X-X$ and $P^{\prime} X-X$ lie in different maximal connected subsets of $M-X$. For suppose this is not true, and the $\operatorname{arc} P P^{\prime}$ contains a subarc $P Q$, such that for every interior point $X$ of $P Q$, the sets $P X-X$ and $P^{\prime} X-X$ lie in the same connected subset of $M-X$. Then, for each point $X$, there is an $\operatorname{arc}$ in $M-X$ joining $P$ to $Q$, and this arc contains as a subset an $\operatorname{arc} A B$, which has only its end points in common with $P Q$, and which is such that $A$ is either $Q$ or an interior point of the $\operatorname{arc} Q X$, and such that $B$ is an interior point of the arc $X P$. Also, the point $P$ is not a limit point of the collection of maximal connected subsets of $M-P Q$ that have limit points on $X Q$, because $P$ is not a limit point of any one of them (since it has property 1 ), and only a finite number of these sets can be of diameter greater than half the distance from $P$ to the nearest point of $X Q{ }^{*}$ Therefore there is a last point (necessarily different from $P$ ) on the $\operatorname{arc} X P$ to which an $\operatorname{arc} A B$, of the type described above, can be constructed. We have therefore shown that corresponding to any point $X$, an arc $A B$ as described above can be constructed having the additional property that no interior point of the $\operatorname{arc} B P$ can be joined to a point of $X Q-X$ by an arc having only its end points in common with $P Q$.

Let us then select a point $B_{0}$, which is an interior point of the $\operatorname{arc} P Q$. Let $A_{1} B_{1}$ be an arc corresponding to $B_{0}$. The point $A_{1}$ is a point of $B_{0} Q-B_{0}$, and $B_{1}$ is an interior point of $P B_{0}$. Let $A_{2} B_{2}$ be an arc corresponding to $B_{1}$. The point $A_{2}$ is a point of $B_{1} B_{0}-B_{1}$, and $B_{2}$ is an interior point of $P B_{1}$. Continuing this process, for $n \geqq 2$, there exists an arc $A_{n+1} B_{n+1}$ corresponding to $B_{n}$, where $A_{n+1}$ is a point of $B_{n} B_{n-1}-B_{n}$, and $B_{n+1}$ is an interior point of $P B_{n}$.

Any set of points $B_{0}, B_{1}, B_{2}, \cdots$ on an arc $P Q$, and such that $B_{i}$ follows $B_{i+1}$, must have a sequential limit point on $P Q$. We shall show that under the given conditions, this sequential limit point must be the point $P$. For suppose a sequence of this type has a point $C$ different from $P$ as a sequential limit point. Then there exists an arc $A^{\prime} B^{\prime}$ corresponding to $C$, where $A^{\prime}$ is a point of $C Q-C$, and $B^{\prime}$ is an interior point of $P C$. Some point of the sequence $B_{0}, B_{1}, B_{2}, \cdots$, say $B_{n}$, is an interior point of the $\operatorname{arc} C A^{\prime}$. By our method of constructing the arc $A_{n+1} B_{n+1}$ corresponding to $B_{n}$, no interior point of $B_{n+1} P$ can be joined to a point of $B_{n} Q-B_{n}$ by an arc having only its end points in common with $P Q$. But $B_{n+1}$ lies between $C$ and $A^{\prime}$, and therefore

* W. L. Ayres, loc. cit., Theorem 1, p. 396. 
$B^{\prime}$ is an interior point of $B_{n+1} P$, while $A^{\prime}$ is a point of $B_{n} Q-B_{n}$. The existence of the $\operatorname{arc} A^{\prime} B^{\prime}$ shows that our given method of construction was not followed in constructing the arc $A_{n+1} B_{n+1}$. Having arrived at this contradiction by supposing $C$ to be the limit of the sequence, it follows that $P$ is the sequential limit point of any sequence $B_{0}, B_{1}, B_{2}, \ldots$, obtained as described above.

The continuum consisting of the $\operatorname{arc} P Q$ and the sequence of $\operatorname{arcs} A_{1} B_{1}$, $A_{2} B_{2}, A_{8} B_{3}, \cdots$ is a continuous curve, every subcontinuum of which is a continuous curve.* Let $M_{1}$ denote the continuum consisting of $P$, the arcs $A_{2 n+1} B_{2 n+1}(n=0,1,2, \cdots)$, and the $\operatorname{arcs} B_{2 n+1} A_{2 n+3}(n=0,1,2, \cdots)$ of the arc $P Q$. Let $M_{2}$ denote the continuum consisting of $P$, the arcs $A_{2 n} B_{2 n}(n=1,2,3, \cdots)$, and the $\operatorname{arcs} B_{2 n} A_{2 n+2}(n=1,2,3, \cdots)$ of the arc $P Q$. These two continua have only the point $P$ in common, and since each is a continuous curve, we can construct in each an arc having $P$ as an end point. But in that case $P$ fails to have property 1 , which is contrary to hypothesis. We have thus established that if $P$ has property 1 , any arc $P P^{\prime}$ contains a set of cut points of $M$ having $P$ as a limit point, each of these points $X$ being such that $P X-X$ and $P^{\prime} X-X$ lie in different maximal connected subsets of $M-X$.

Let $P P^{\prime}$ be an arc in $M$, and let $P_{1}, P_{2}, P_{3}, \cdots$ be a sequence of points of $P P^{\prime}$ which are cut points of $M$ of the type described above, and whose sequential limit point is $P$. Given any positive number $\epsilon_{1}$, we can select a positive number $\epsilon$, such that $\epsilon$ is less than $\epsilon_{1}$, and is less than the distance from $P$ to $P^{\prime}$. The number of maximal connected subsets of $M-P P^{\prime}$ of diameter greater than $\epsilon / 6$ is finite, and since $P$ is not a limit point of any one of these sets, we can select an integer $n$ such that the diameter of $P P_{n}$ is less than $\epsilon / 6$, and such that no maximal connected subset of $M-P P^{\prime}$ of diameter greater than $\epsilon / 6$ has any limit points on $P P_{n}$. If we denote by $N$ the maximal connected subset of $M-P_{n}$ that contains $P$, it follows that the diameter of $N$ is less than $\epsilon / 2$. The continuum $N+P_{n}$ cannot contain a simple closed curve enclosing the set $P_{n} P^{\prime}-P_{n}$, otherwise the diameter of $N$ would be greater than the distance from $P$ to $P^{\prime}$, which is impossible.

If no simple closed curve in $M-N$ encloses a point of $N$, then if we add to $N+P_{n}$ all points of the plane which are interior to a simple closed curve in $N+P_{n}$, we obtain a continuum $K$ which does not separate the plane. Let $H_{1}$ denote the continuum consisting of all points of $M+K-\left(K-P_{n}\right)$, and let $B$ denote the continuum consisting of $H_{1}$ and all points of the plane which are interior to a simple closed curve in $H_{1}$. The two continua $K$ and $H$

* H. M. Gehman, Some conditions under which a continuum is a continuous curve, Annals of Mathematics, (2), vol. 27 (1926), pp. 381-384. See especially Theorem 2, p. 382. 
satisfy certain conditions* under which there exists a simple closed curve enclosing $K-P_{n}$ but not enclosing any other points of $K+H$, and containing $P_{n}$ but not containing any other points of $K+H$. Therefore there exists a simple closed curve enclosing $N$, not enclosing $P_{n} P^{\prime}-P_{n}$, and having only $P_{n}$ in common with $M$.

In case a simple closed curve in $M-N$ encloses a point of $N$, it encloses all of $N$. Then, by Theorem 3 of S.P.S., there exists a simple closed curve having the properties mentioned at the end of the preceding paragraph.

Let us denote by $J$ the simple closed curve enclosing $N$. In case $J$ is of diameter less than $\epsilon$, its interior is the domain required in order that $P$ have property 7. In case $J$ is of diameter greater than $\epsilon$, we shall show how to replace $J$ by a simple closed curve whose diameter is less than $\epsilon$, which encloses $N$, and which has only $P_{n}$ in common with $M$. Let us denote by $I$ the interior of $J$.

Let us denote by $K$, the continuum consisting of all points of $M$ in $J+I$, and all points of the plane which are interior to a simple closed curve of $M$ in $J+I$. Then by Theorem 1 of S.P.S., there exists a simple closed curve $L$ which encloses $K$ and is such that every point of $L$ plus its interior is at a distance less than $\epsilon / 4$ from some point of $K$. By the way in which the point $P_{n}$ was selected, the diameter of $K$ is less than $\epsilon / 2$, and therefore the diameter of $L$ is less than $\epsilon$. Since the diameter of $J$ is greater than $\epsilon, J$ is not entirely contained in $L$ plus its interior, and therefore $L$ contains some points which are interior to $J$. The two simple closed curves $J$ and $L$ satisfy the conditionst under which there exists a simple closed curve $J^{\prime}$ which is a subset of $J+L$, which contains an $\operatorname{arc}$ through $P_{n}$, and every point of whose interior is interior to both $J$ and $L$. The simple closed curve $J^{\prime}$ has only $P_{n}$ in common with $M$, because $J^{\prime}$ is a subset of $J$ plus that portion of $L$ which is interior to $J$, and this portion of $L$ has no points in common with $M$. Furthermore, since every point of the interior of $J^{\prime}$ is a point of the interior of $L$, the diameter of $J^{\prime}$ cannot be greater than the diameter of $L$, that is, the diameter of $J^{\prime}$ is less than $\epsilon$. The existence of the simple closed curve $J^{\prime}$ shows that $P$ has property 7 , as the interior of $J^{\prime}$ is the domain required in order that $P$ have property 7 . This completes the proof of Theorem 1.

Definitron. A point of a continuous curve which has properties 1-7 is said to be an end point of the continuous curve.

- R. L. Moore, Concerning the separation of point sets by curves, Proceedings of the National Academy of Sciences, vol. 11 (1925), pp. 469-476. See Theorem 2, p. 470. We shall refer to this paper hereafter as S.P.S.

$\dagger$ R. L. Moore, On the Lie-Riemann-Helmholtz-Hilbert problem of the foundations of geometry, American Journal of Mathematics, vol. 41 (1919), pp. 299-319. See especially Theorem 26, p. 311. 
Note that in the course of the proof of Theorem 1, we have also proved the following theorem:

Theorem 2. An end point $P$ of a continuous curve $M$ has this property: given any positive number $\epsilon$, there exists a simple closed curve enclosing $P$, of diameter less than $\epsilon$, and having just one point in common with $M$. Conversely, if a point $P$ of a continuous curve $M$ has the above property, then $P$ is an end point of $M$.

\section{CONCERNING PROPERTIES 8-9 FOR A CONTINUOUS CURVE}

We shall now introduce two additional properties of a point $P$ of a continuous curve $M$.

Property 8. If $P^{\prime}$ and $P^{\prime \prime}$ are any two points of $M$ different from $P$, then any two subcontinua of $M$ irreducible between $P$ and $P^{\prime}$, and between $P$ and $P^{\prime \prime}$, respectively, have in common a continuum containing $P$.

Property 9. There exists a positive number $x$, such that if $P^{\prime}$ and $P^{\prime \prime}$ are any two points of $M$ at a distance less than $x$ from $P$, then one of any two subcontinua of $M$ irreducible between $P$ and $P^{\prime}$, and between $P$ and $P^{\prime \prime}$, respectively, contains the other.

Properties 8 and 9 are due to Yoneyama.* A property analogous to property 9 has also been given by Young. $\dagger$

In Part 4 of this paper, it is shown that if $M$ is any bounded continuum, and $P$ has property 9 , it has property 8 ; and if $P$ has property 8 , it has property 4 . Therefore, in the case of a continuous curve, if $P$ has property 8 , it has properties 1-7. The following examples will show (1) that a point $P$ of a continuous curve $M$ may have properties 1-7, without having properties 8 or 9 ; (2) that a point $P$ of a continuous curve $M$ may have properties $1-8$, without having property 9.

Example 1. Let $M$ consist of the point $(0,0)$, and the circles with center at $\left(3 / 2^{n}, 0\right)$ and with radius equal to $1 / 2^{n}$, for $n=1,2,3, \cdots$, and let $P$ be the point $(0,0)$. The point $P$ has properties $1-7$, but not properties 8 and 9.

Example 2. Let $M$ consist of the straight line intervals between $(0,0)$ and $(1,0)$, and between $\left(1 / 2^{n}, 0\right)$ and $\left(1 / 2^{n}, 1 / 2^{n}\right)$, for $n=1,2,3, \cdots$, and let $P$ be the point $(0,0)$. The point $P$ has properties $1-8$, but not property 9.

* K. Yoneyama, On continuous set (sic) of points, II, Tohoku Mathematical Journal, vol. 18 (1920), p. 254.

† W. H. Young and G. C. Young, The Theory of Sets of Points, 1906, p. 220. 
Theorem 3. A necessary and sufficient condition that a point $P$ of a continuous curve $M$ have property 8 , is that $P$ have properties 1-7, and that any arc $P Q$ of $M$ contains a sub-arc $P X$, every interior point of which is a cut point of $M$.

Let $P$ be an end point of a continuous curve $M$ such that if $P Q$ is any arc of $M$, then $P Q$ contains a sub-arc $P X$ every interior point of which is a cut point of $M$. This is equivalent to the statement that if $P Q$ is any arc of $M$, the point $P$ is not a limit point of those maximal connected subsets of $M-P Q$ that have more than one limit point on $P Q$. We shall now show that $P$ also has property 8 .

Let us select a definite point $Q$ and a definite $\operatorname{arc} P Q$. Let $\epsilon$ be a positive number which is less than the distance from $P$ to $Q$, and less than the distance from $P$ to any point of a maximal connected subset of $M-P Q$ that has more than one limit point on $P Q$. By Theorem 2, we can construct a simple closed curve $J$ enclosing $P$, of diameter less than $\epsilon$, and having just one point $X$ in common with $M$. The point $X$ is necessarily an interior point of the arc $P Q$.

If $P^{\prime}$ is any point exterior to $J$, any subcontinuum of $M$ which is irreducible between $P$ and $P^{\prime}$ must contain $X$, and therefore can be expressed as the sum of two continua irreducible between $P^{\prime}$ and $X$ and between $X$ and $P$, respectively, and having only $X$ in common. We shall now show that any subcontinuum of $M$ irreducible between $P$ and any point $Y$ lying in $J$ plus its interior, has an arc in common with the arc $P X$ of the arc $P Q$.

Note that under our given conditions, every interior point of the arc $P X$ is a cut point of $M$. Therefore if $Y$ is any point of $P X-P$, any connected subset of $M$ containing $Y$ and $P$ necessarily contains all points of the $\operatorname{arc} P Y$, and therefore any subcontinuum of $M$ irreducible between $P$ and $Y$ must coincide with this arc. If $Y$ is a point of a maximal connected subset of $M-P Q$ in the interior of $J$, any connected subset of $M$ containing $Y$ and $P$ must contain the point $Z$ which is the limit point on $P Q$ of the maximal connected subset of $M-P Q$ that contains $Y$. Since $P$ has properties 1-7, $Z$ is different from $P$. As before, any connected subset of $M$ that contains $Z$ and $P$ must contain the $\operatorname{arc} P Z$, and therefore any subcontinuum of $M$ irreducible between $P$ and $Y$ has the arc $P Z$ in common with $P Q$.

Therefore if $P^{\prime}$ and $P^{\prime \prime}$ are any two points of $M$, each one of any two subcontinua of $M$ irreducible between $P$ and $P^{\prime}$, and between $P$ and $P^{\prime \prime}$ respectively, has an arc containing $P$ in common with $P Q$, and the common part of these two arcs is the continuum required in order that $P$ have property 8 . Therefore the condition is sufficient. 
Suppose now that a continuous curve $M$ contains a point $P$ which has properties 1-8, and suppose that for some arc $P Q$, the point $P$ is a limit point of non-cut points of $M$ on $P Q$, and is therefore also a limit point of maximal connected subsets of $M-P Q$ which have more than one limit point on $P Q$. Let us select a sequence $D_{1}, D_{2}, D_{3}, \cdots$ of maximal connected subsets of $M-P Q$ having $P$ as a limit point, such that $D_{i}$ has at least two limit points on $P Q$, and such that every limit point of $D_{i+1}$ on $P Q$ lies between $P$ and each limit point of $D_{i}$ on $P Q$. The sequence can be selected so as to satisfy this latter condition, because $P$ is not a limit point of any one of the sets $D_{i}$ (because $P$ has properties 1-8), and because the number of maximal connected subsets of $M-P Q$ of diameter greater than any given positive number is finite.

If $A_{i}$ and $B_{i}$ are two points of $P Q$ which are limit points of $D_{i}$, an arc can be constructed from $A_{i}$ to $B_{i}$ in the set $D_{i}+A_{i}+B_{i}$. The set of arcs $A_{i} B_{i}(i=1,2,3, \cdots)$ plus the set of $\operatorname{arcs} B_{i} A_{i+1}(i=1,2,3, \cdots)$ of the arc $P Q$, plus the point $P$, is a continuous curve, by the argument given in the proof of Theorem 1, and therefore this set contains an arc from $A_{1}$ to $P$. However this arc $A_{1} P$ and the $\operatorname{arc} P Q$ do not have in common a continuum containing $P$, and therefore $P$ fails to have property 8 , which is contrary to hypothesis. Therefore the condition is necessary.

Corollary 3a. If a point $P$ of an acyclic* continuous curve has any one of the properties 1-8, it has all the others.

Theorem 4. A necessary and sufficient condition that a point $P$ of a continuous curve $M$ have property 9 , is that if $P Q$ is, any arc of $M$, then $P$ is not a limit point of $M-P Q$.

Let $P$ be a point having the given property, and let us select a definite $\operatorname{arc} P Q$ of $M$. Then since $P$ is not a limit point of $M-P Q$, there exists a point $Y$ of $P Q$, such that no point of the arc $P Y$ is a limit point of $M-P Q$. Let $x$ be a positive number which is less than the distance from $P$ to any point of $M-P Y$. If $P^{\prime}$ is any point of $M$ at a distance less than $x$ from $P$, the point $P^{\prime}$ is a point of the $\operatorname{arc} P Y$. Any connected subset of $M$ containing both $P$ and $P^{\prime}$ must contain the arc $P P^{\prime}$, and therefore the only subcontinuum of $M$ which is irreducible between $P$ and $P^{\prime}$ is the $\operatorname{arc} P P^{\prime}$. Therefore if $P^{\prime}$ and $P^{\prime \prime}$ are any two points of $M$ at a distance less than $x$ from $P$, one of the two arcs $P P^{\prime}, P P^{\prime \prime}$ will contain the other, and therefore $P$ has property 9 , and the condition is sufficient.

\footnotetext{
* An acyclic continuous curve is a continuous curve containing no simple closed curve.
} 
The condition is necessary, for suppose $P$ has property 9 (and therefore properties 1-8), and yet there is an $\operatorname{arc} P Q$ of $M$ such that $P$ is a limit point of $M-P Q$. Then if we select any positive number $x$, there are points of two different maximal connected subsets of $M-P Q$ at a distance less than $x$ from $P$, for if there were only one, $P$ would fail to have properties 1-9. Let these sets be $D_{1}$ and $D_{2}$. If $P_{i}$ is a point of $D_{i}$, an $\operatorname{arc} P_{i} P$ can be constructed in $D_{i}+P Q$ and evidently neither of the $\operatorname{arcs} P_{1} P, P_{2} P$ contains the other, contrary to our hypothesis that $P$ has property 9 .

CoRollary 4a. If a point $P$ of an arc has any one of the properties 1-9, it has all the others.

To recapitulate:

ThEOREM 5. For a point of a continuous curve, properties 1-7, Whyburn's property, Ayres' property, and the property mentioned in Theorem 2 are equivalent; property 8 is stronger than any of these; and property 9 is stronger than property 8. For a point of an acyclic continuous curve, properties 1-8 are equivalent, and property 9 is stronger than any of them. For a point of an arc, properties 1-9 are equivalent.

\section{ConCerning PROPERTIES 4-9 FOR A BoUnded CONTINUUM}

In this part, we shall consider the logical relations between properties 4-9, for the case where $M$ is bounded continuum.

Theorem 6. If a point $P$ of a bounded continuum $M$ has property 9, it has property 8.

Suppose $P$ has property 9 , but not property 8 . Then there exist two points $P^{\prime}$ and $P^{\prime \prime}$ of $M$, and two subcontinua $N^{\prime}, N^{\prime \prime}$ of $M$ irreducible between $P$ and $P^{\prime}$, and between $P$ and $P^{\prime \prime}$, respectively, but which do not have in common any continuum containing $P$. Since $P$ has property 9 , we can select a point $Q^{\prime}$ of $N^{\prime}$, and a point $Q^{\prime \prime}$ of $N^{\prime \prime}$ sufficiently close to $P$ that one of any two subcontinua of $M$ irreducible between $P$ and $Q^{\prime}$, and between $P$ and $Q^{\prime \prime}$, contains the other. Since $Q^{\prime}$ and $P$ are points of $N^{\prime}$, the set $N^{\prime}$ contains a subcontinuum $K^{\prime}$ which is irreducible between $Q^{\prime}$ and $P$, and similarly $N^{\prime \prime}$ contains a subcontinuum $K^{\prime \prime}$ which is irreducible between $Q^{\prime \prime}$ and $P$. We have shown above that one of the two continua $K^{\prime}, K^{\prime \prime}$ contains the other. Suppose $K^{\prime}$ contains $K^{\prime \prime}$. If so, the two continua $N^{\prime}$ and $N^{\prime \prime}$ have in common the continuum $K^{\prime \prime}$ which contains $P$, which is contrary to our supposition that $N^{\prime}$ and $N^{\prime \prime}$ do not have in common a continuum containing $P$. 
Theorem 7. If a point $P$ of a bounded continuum $M$ has property 8, it has property 4.

Suppose $P$ has property 8 , but not property 4 . Then, $M$ contains a subcontinuum $N$ containing $P$, such that $N-P$ is not connected. Let $H_{1}+H_{2}$ be any method of expressing $M_{1}-P$ as the sum of two sets having no points in common and neither containing a limit point of the other. Then $H_{1}+P$ and $H_{2}+P$ are two subcontinua of $M$ having only $P$ in common. The set $H_{1}+P$ contains a subcontinuum irreducible between $P$ and any point $P^{\prime}$ of $H_{1}+P$, and $H_{2}+P$ contains a subcontinuum irreducible between $P$ and any point $P^{\prime \prime}$ of $H_{2}+P$. These two continua have only $P$ in common, and therefore $P$ does not have property 8 , contrary to hypothesis.

Theorem 8. If a point $P$ of a bounded continuum $M$ has property 7 , it has property 6.

Suppose $P$ has property 7 , but not property 6 . Then there is some subcontinuum $N$ of $M$ containing $P$, such that $P$ is a limit point of some connected set $L$ which is a subset of $M-N$. Let $X$ be a point of $N-P$, and $Y$ a point of $L$. Let $\epsilon$ be less than the distance from $P$ to $X$, and less than the distance from $P$ to $Y$. Since $P$ has property 7 , there exists a domain $D$ which contains $P$, whose exterior contains both $X$ and $Y$, and whose boundary has only one point $Q$ in common with $M$. The connected set $N$ contains the point $P$ in $D$, and the point $X$ exterior to $D$, and therefore contains a point of the boundary of $D$. Therefore $Q$ is a point of $N$. But the connected set $L+P$ also contains the point $P$ in $D$, and the point $Y$ exterior to $D$, and therefore $Q$ is also a point of $M-N$. But it is impossible for $Q$ to be both a point of $N$ and a point of $M-N$, and therefore if $P$ has property 7 , it also has property 6 .

Theorem 9. If a point $P$ of a bounded continuum $M$ has property 6 , it has property 5.

Suppose $P$ has property 6 , but not property 5 . Then $M$ contains a subcontinuum $N$ containing $P$, which is irreducible between two points $P^{\prime}$, $P^{\prime \prime}$ different from $P$. Let $K$ be any subcontinuum of $N$ containing $P$, but not $P^{\prime}$ or $P^{\prime \prime}$, and let $D^{\prime}$ be the maximal connected subset of $N-K$ that contains $P^{\prime}$. Since $P$ has property $6, P$ is not a limit point of $D^{\prime}$, and therefore if $D^{\prime}$ also contained $P^{\prime \prime}$, the continuum consisting of $D^{\prime}$ and its limit points in $K$ would be a proper subcontinuum of $N$ containing $P^{\prime}$ and $P^{\prime \prime}$, which is impossible. Therefore $P^{\prime \prime}$ lies in a maximal connected subset of $N-K$ different from $D^{\prime}$, and we shall denote the one in which $P^{\prime \prime}$ lies by $D^{\prime \prime}$. The set $K+D^{\prime}+D^{\prime \prime}$ is a continuum which is a subset of $N$ and contains $P^{\prime}$ and $P^{\prime \prime}$, 
and must therefore be identical with $N$. Therefore $N-K$ consists of two and only two maximal connected subsets, one of which contains $P^{\prime}$, and the other $P^{\prime \prime}$. Let us denote by $E^{\prime}$ and $E^{\prime \prime}$ the sets of points of $K$ which are limit points of $D^{\prime}$ and $D^{\prime \prime}$ respectively. Since $P$ is not a point of either $E^{\prime}$ or $E^{\prime \prime}$, the sets $E^{\prime}, E^{\prime \prime}$ have no points in common. For if they had points in common, $D^{\prime}+E^{\prime}+D^{\prime \prime}+E^{\prime \prime}$ would be a proper subcontinuum of $N$ containing both $P^{\prime}$ and $P^{\prime \prime}$, which is impossible. Also $D^{\prime}+E^{\prime}$ is irreducible between $P^{\prime}$ and any point of $E^{\prime}$, and $D^{\prime \prime}+E^{\prime \prime}$ is irreducible between $P^{\prime \prime}$ and any point of $E^{\prime \prime}$, while $K$ is irreducible between some point of $E^{\prime}$ and some point of $E^{\prime \prime}$.

Let us select a sequence of subcontinua of $N: N_{1}, N_{2}, N_{3}, \cdots$, such that for $i=1,2,3, \cdots$, (1) $N_{i}$ contains $N_{i+1}$, (2) $N_{i}$ contains $P$, but not $P^{\prime}$ or $P^{\prime \prime}$, (3) the diameter of $N_{i}$ is less than $1 / i$. For each of the sets $N_{i}$, let $D_{i}^{\prime}$ and $D_{i}^{\prime \prime}$ denote the maximal connected subsets of $N-N_{i}$ containing $P^{\prime}$ and $P^{\prime \prime}$ respectively. The sequence of continua $N_{1}+D_{1}^{\prime}, N_{2}+D_{2}^{\prime}, N_{3}+D_{3}^{\prime}$, $\cdots$, has the property that each continuum contains the one following it in the sequence. There is therefore a continuum $K^{\prime}$ common to the members of the sequence. Evidently $K^{\prime}$ contains $P^{\prime}$ and $P$, but. contains no points of any of the sets $D_{i}^{\prime \prime}$.

Also there is a continuum $K^{\prime \prime}$ common to all the members of the sequence $N_{1}+D_{1}^{\prime \prime}, N_{2}+D_{2}^{\prime \prime}, N_{3}+D_{3}^{\prime \prime}, \cdots$, and $K^{\prime \prime}$ contains $P^{\prime \prime}$ and $P$, but no points of any of the sets $D_{i}^{\prime}$. Therefore the only points that $K^{\prime}$ and $K^{\prime \prime}$ can have in common are those points which are common to all members of the sequence $N_{1}, N_{2}, N_{3}, \cdots$, and the point $P$ is the only point common to all the sets $N_{i}$. Therefore $K^{\prime}$ and $K^{\prime \prime}$ have only $P$ in common.

The set $K^{\prime}$ can also be expressed as the point $P$ plus the sum of the infinite collection of connected sets $D_{1}^{\prime}, D_{2}^{\prime}, D_{3}^{\prime}, \cdots$, each of which is contained in all that follow it in the sequence. It therefore follows that $P$ is a non-cut point of $K^{\prime}$, and that therefore the point $P$, considered as a point of the continuum $K^{\prime \prime}$, is a limit point of the connected subset $K^{\prime}-P$ of $M-K^{\prime \prime}$. That is, $P$ fails to have property 6 , which is contrary to hypothesis.

THEOREM 10. If a point $P$ of a bounded continuum $M$ has property 5 , it has property 4.

Suppose $P$ has property 5 , but not property 4 . Then $M$ contains a subcontinuum $N$, such that $N-P$ is disconnected. In that case, $N$ can be expressed as the sum of two continua $N_{1}$ and $N_{2}$ having only $P$ in common. Let $P_{i}$ be a point of $N_{i}(i=1,2)$, and let $K_{i}$ be a subcontinuum of $N_{i}$ which is irreducible between $P_{i}$ and $P$. The continuum $K_{1}+K_{2}$ is irreducible between $P_{1}$ and $P_{2}$, and therefore $P$ does not have property 5 , which is contrary to hypothesis. 
Theorem 11. If a point $P$ of a bounded continuum $M$ has both property 5 and property 9, it has property 6.

We shall show that if $P$ has properties 5 and 9 , and if $N$ is any subcontinuum of $M$ containing $P$, then $P$ is not a limit point of the set $M-N$, and therefore $P$ has property 6 . Suppose then that $P$ has properties 5 and 9 , but that for some subcontinuum $N$ of $M$, the point $P$ is a limit point of $M-N$. We shall show that this leads to a contradiction.

Let $P_{0}$ be a point of $N$ whose distance from $P$ is less than the distance $x$ (of property 9). Then $N$ contains a subcontinuum $N_{0}$ irreducible between $P_{0}$ and $P$, and $P$ is also a limit point of the set $M-N_{0}$. If $P^{\prime}$ is any point of $M-N_{0}$ whose distance from $P$ is less than $x$, every subcontinuum of $M$ that contains $P^{\prime}$ and $P$ must contain the continuum $N_{0}$, otherwise $P$ fails to have property 9 .

Let $P_{1}$ be a point of $M-N_{0}$ whose distance from $P$ is less than $x$, and let $N_{1}$ be a subcontinuum of $M$ irreducible between $P_{1}$ and $P$. We have just shown above that $N_{0}$ is a subset of $N_{1}$. Since $N_{0}$ contains $P$, the set $N_{1}-N_{0}$ is connected.

If $N_{1}$ contains all points of $M-N_{0}$ which are at a distance from $P$ less than some constant $k$ then if we add to $N_{1}-N_{0}$ the set of its limit points (which includes $P$ ), the resulting continuum must contain $N_{0}$, as we have shown above. In other words, $N_{0}$ is a continuum of condensation of $N_{1}$. But in that case $N_{1}$ is irreducible between $P_{1}$ and any point of $N_{0}$, and $P$ fails to have property 5 . Therefore there are points of $M-N_{0}$ arbitrarily close to $P$ which are not points of $N_{1}$, that is, $P$ is a limit point of the set $M-N_{1}$.

Therefore let us select a point $P_{2}$ of $M-N_{1}$ whose distance from $P$ is less than $x / 2$. If $N_{2}$ is any subcontinuum of $M$ irreducible between $P_{2}$ and $P$, then $N_{2}$ contains $N_{1}$ and $P$ is a limit point of the set $M-N_{2}$. In general, if (for $i=2,3,4, \cdots$ ) $P_{i}$ is a point of $M-N_{i-1}$ whose distance from $P$ is less than $x / i$, then any subcontinuum $N_{i}$ of $M$ which is irreducible between $P_{i}$ and $P$, contains $N_{i-1}$, and $P$ is a limit point of the set $M-N_{i}$.

Let us then select a definite sequence of points $P_{0}, P_{1}, P_{2}, \cdots$, and a definite sequence of continua $N_{0}, N_{1}, N_{2}, \cdots$ having the properties described above. We shall show that if $K$ denotes the continuum consisting of $N_{0}+N_{1}+N_{2}+\cdots$ plus limit points, then every proper subcontinuum of $K$ is a continuum of condensation of $K$ and therefore $K$ is indecomposable.*

\footnotetext{
* A continuum is said to be indecomposable if it cannot be expressed as the sum of two of its proper subcontinua.
} 
Suppose some proper subcontinuum $L$ of $K$ is not a continuum of condensation of $K$. Evidently the points of $L$ which are not limit points of $K-L$ do not lie entirely in the set of limit points of $N_{0}+N_{1}+N_{2}+\cdots$, and therefore $L$ must contain a point $Q$ of one of the sets $N_{0}, N_{1}, N_{2}, \cdots$, say $N_{i}$, such that $Q$ is not a limit point of $K-L$.

Suppose $L$ does not contain $P$. The closed set $H$ consisting of $(K-L)$ plus limit points of $(K-L)$ is a proper subset of $K$, and consists of a collection $(G)$ of maximal continua of $B$. The continuum $G_{1}$ of this collection that contains $P$ can contain none of the points $P_{j}+P_{j+1}+P_{j+2}+\cdots$. The continuum $L$ also can contain only a finite number of points of the sequence $P_{0}, P_{1}, P_{2}, \cdots$ and therefore there exists an integer $k$ such that no point of the sequence $P_{k}, P_{k+1}, P_{k+2}, \cdots$ is a point of $L$ or of $G_{1}$. Let $P_{m}$ and $P_{n}$ be two points such that $m \geqq k, n \geqq k$, and $m \geqq j, n \geqq j$. If $P_{m}$ and $P_{n}$ were in different continua of $(G)$, then by adding to $L+G_{1}$ each of these continua in turn, we obtain two continua in which we can construct subcontinua of $M$ irreducible between $P_{m}$ and $P$, and between $P_{n}$ and $P$ respectively, neither of which contains the other, which is contrary to the hypothesis that $P$ has property 9 . Therefore all points of $P_{j+k}, P_{j+k+1}$, $P_{j+k+2}, \cdots$ are points of the same maximal continuum of $H$. Since $P$ is a limit point of the set $P_{j+k}+P_{j+k+1}+P_{j+k+2}+\cdots$, it follows that $P$ also belongs to the maximal continuum of $H$ that contains this set. But this is impossible, as the continuum $G_{1}$ containing $P$ contains no points of the set $P_{j}+P_{j+1}+P_{j+2}+\cdots$.

Suppose $L$ contains $P$. If $L$ contains any point $P_{n}$ of the set $P_{0}+P_{1}+P_{2}$ $+\cdots$, then $L$ must contain all the points $P_{0}+P_{1}+\cdots+P_{n}$. If there were no integer $k$ such that $P_{k}$ is a point of $K-L$ then $L$ would be identical with $K$, which is contrary to our supposition that $L$ is a proper subcontinuum of $K$. Therefore there is some integer $k$, such that $P_{k}, P_{k+1}, P_{k+2}, \cdots$ are all points of $K-L$. As in the preceding paragraph, all the points $P_{k}, P_{k+1}$, $P_{k+2}, \cdots$ lie in the same continuum of $(G)$, and this continuum also contains $P$. But this is impossible, as the continuum containing $P$ cannot contain any points of the sequence $P_{i}+P_{j+1}+P_{j+2}+\cdots$. Therefore $K$ is indecomposable.

Any indecomposable continuum $K$ has this property: given any point $P$ there are two other points such that $K$ is irreducible between any two points of the three. It follows that if $M$ contains an indecomposable continuum $K$ containing $P$, then $P$ does not have property 5 . But this is contrary to hypothesis, and therefore Theorem 11 is true.

Note that the above argument establishes the following theorem: 
Theorem 12. Given a sequence of points $P_{0}, P_{1}, P_{2}, \cdots$ whose sequential limit point is $P$, and a collection of continua $N_{0}, N_{1}, N_{2}, \cdots$, such that $N_{i}$ is irreducible between $P_{i}$ and $P$, and such that $N_{i}$ is a proper subset of $N_{i+1}$. If the continuum $K$ consisting of $N_{0}+N_{1}+N_{2}+\cdots$ plus limit points has the property that any subcontinuum of $K$ irreducible between $P_{i}$ and $P$ is a subset of every subcontinuum of $K$ irreducible between $P_{i+1}$ and $P$, then $K$ is an indecomposable continuum.

This theorem can be used to establish the indecomposability of two examples due to Knaster.* To recapitulate:

ThEOREM 13. For a point of a bounded continuum,

(1) property 9 is stronger than property 8;

(2) property 8 is stronger than property 4;

(3) property 7 is stronger than property 6 ;

(4) property 6 is stronger than property 5;

(5) property 5 is stronger than property 4;

(6) properties 5 and 9 together are stronger than property 6.

The following set of examples shows that no logical relations exist among properties 4-9, excepting those given in Theorems 6-11, and therefore the truth of Theorem 13 follows from the examples and from Theorems 6-11.

An end point of a straight line interval has all the properties 4-9; an interior point has none of them. The point $P$ of example 1 (of Part 3 ) has properties 4-7, but not 8 or 9 . The point $P$ of example 2 has properties 4-8, but not 9 .

EXample 3. Let $M$ consist of the curve $y=\sin (1 / x)$, for $0<x \leqq 1$, and the straight line interval from $(0,-1)$ to $(0,1)$. Let $P$ be the point $(0,1)$, which has properties 4,8 , and 9 , but not 5,6 , or 7 .

Example 4. Let $M$ consist of the continuum of example 3, plus the curve $y=\sin (1 / x)$, for $0>x \geqq-1$. Let $P$ be the point $(0,1)$, which has properties 4 and 8 , but none of the properties 5-7, 9.

ExAmple 5. Let $M$ consist of the continuum of example 3, plus the set of semicircles lying on the negative side of the $y$-axis, with center at the point $\left(0,1-3 / 2^{n}\right)$ and radius equal to $1 / 2^{n}$, for $n=1,2,3, \cdots$. Let $P$ be the point $(0,1)$, which has property 4 , but none of the properties 5-9.

EXample 6. Let $M$ consist of the continuum of example 2, plus the set of curves

- Contained in C. Kuratowski, Theorie des continus irréductibles entre deux points, Fundamenta Mathematicae, vol. 3 (1922), pp. 200-223. These examples are described on pp. 209-210 and on pp. 216-217 respectively. 


$$
y=\frac{1}{2^{n+1}}+\left(\frac{1}{2^{n}}-\frac{x}{2}\right) \sin \left(\frac{1}{x-1 / 2^{n}}\right),
$$

between $\left(1 / 2^{n}\right)<x \leqq\left(1 / 2^{n-1}\right)$, for $n=1,2,3, \cdots$. Let $P$ be the point $(0,0)$, which has properties 4 and 5, but none of the properties 6-9.

ExAMPLE 7. Let $M$ consist of the straight line intervals from $(1,0)$ to $(0,0)$, and from $(1,0)$ to $(0,1 / n)$, for $n=1,2,3, \cdots$. Let $P$ be the point $(0,0)$, which has properties 4,5 , and 8 , but none of the properties $6,7,9$.

EXAMPLE 8.* Let $M$ consist of the straight line intervals from $(0,0)$ to $(1,0)$, from $\left(1 / 2^{n}, 0\right)$ to $\left(1 / 2^{n}, 1 / 2^{n}\right)$, from $\left(1 / 2^{n}, 1 / 2^{n}\right)$ to $\left(-1 / 2^{n}, 1 / 2^{n}\right)$, from $\left(-1 / 2^{n}, 1 / 2^{n}\right)$ to $\left(-1 / 2^{n},-1 / 2^{n}\right)$, from $\left(-1 / 2^{n},-1 / 2^{n}\right)$ to $\left(1,-1 / 2^{n}\right)$, from $\left(1,-1 / 2^{n}\right)$ to $\left(1,-3 / 2^{n+2}\right)$, from $\left(1,-3 / 2^{n+2}\right)$ to $\left(0,-3 / 2^{n+2}\right)$ for $n=0,1,2, \cdots$ Let $P$ be the point $(0,0)$, which has properties $4,5,6$, and 8 , but not property 7 or property 9 .

EXAMPLE 9. Let $M$ consist of the continuum of example 8, plus the set of semicircles lying on the positive side of the $x$-axis, with center at the point $\left(3 / 2^{n}, 0\right)$ and radius equal to $1 / 2^{n}$, for $n=2,3,4, \cdots$. Let $P$ be the point $(0,0)$, which has properties $4,5,6$, but none of the properties 7,8 , and 9 .

EXAMPLE 10. Let $M_{0}$ denote the indecomposable continuum described by Knaster in his thesis, $\dagger$ and designated there by $K_{1}$. This continuum lies within the square whose vertices are $(0,0),(1,0),(1,1),(1,0)$ and is irreducible between the point $(1,0)$ and any point which cannot be joined to $(1,0)$ by an arc in $M_{0}$. The continuum $M_{0}$ can also be constructed by the method used in constructing the second example in Kuratowski's article.

Let $M_{n}(n=1,2,3, \cdots)$ be the set of points $\left(x^{\prime}, y^{\prime}\right)$ obtained by subjecting the points $(x, y)$ of $M_{0}$ to the following transformation: $x^{\prime}=\left(x+2^{n+1}-2\right) / 2^{n}$, $y^{\prime}=y / 2^{n}$. Let $P$ be the point $(2,0)$, and let $M$ be the continuum consisting of $P+M_{0}+M_{1}+M_{2}+\cdots$ The point $P$ has properties $4,5,6,8$, and 9 , but not property 7 .

EXAMPLE 11. Let $M$ denote an indecomposable continuum every subcontinuum of which is indecomposable. An example of such a continuum has been given by Knaster, $\ddagger$ who designates it by $K_{3}$. If $P$ is any point of $M$, $P$ has properties 4,8, and 9, but none of the properties 5-7. This combination of properties of a point has already been illustrated in example 3 .

\section{CONCERNING PROPERTY 3 FOR A BOUNDED CONTINUUM}

The following theorem follows from the definitions of properties 3 and 6 .

* This example is due to Whyburn, loc. cit., proof of Theorem 30.

† B. Knaster, Un continu dont tout sous-continu est indécomposable, Fundamenta Mathematicae, vol. 3 (1922), pp. 245-286. This example is described on pp. 269-271.

$\ddagger$ Loc. cit., pp. 275-279. 
Theorem 14. If a point $P$ of a bounded continuum $M$ has property 3, it has property 6.

We have not been able to determine whether or not $P$ must have property 3 when it has property 6 . However, the arguments given to prove Theorems 8 and 11 also establish the following theorems concerning property 3 .

Theorem 15. If a point $P$ of a bounded continuum $M$ has property 7, it has property 3 .

Theorem 16. If a point $P$ of a bounded continuum $M$ has property 5 and property 9, it has property 3.

We can combine Theorem 13 and the results of this section into the following theorem.

ThEOREM 17. For a point of a bounded continuum

(1) property 9 is stronger than property 8;

(2) property 8 is stronger than property 4 ;

(3) property 7 is stronger than property 3;

(4) property 6 is stronger than property 5;

(5) property 5 is stronger than property 4;

(6) properties 5 and 9 are stronger than property 3;

(7) either properties 3 and 6 are equivalent, or property 3 is stronger than property 6.

In case property 3 is stronger than property 6 , the question remains whether examples exist of a point having properties 4-6, 8 but not 3, 7, 9, and of a point having properties $4-6$, but not properties $3,7-9$, or whether some further logical relations exist among properties 3-9 for a bounded continuum.

6. SOME THEOREMS CONCERNING POINTS WITH PROPERTIES 1-9

In the preceding discussion we have assumed the following theorem, whose truth follows from the definitions of properties 1-9.

Theorem 18. If a point $P$ of $a$ bounded continuum $M$ has property $x$ (where $x=1,2, \cdots, 9$ ), and $N$ is a subcontinuum of $M$ containing $P$, then $P$ has property $x$ in the continuum $N$.

THEOREM 19. The set of points of a bounded continuum which have property 3 is totally disconnected. 
If the theorem were not true, there would exist a bounded continuum $M$ which contains a connected subset $K$ consisting of more than one point, such that every point of $K$ has property 3 . Let $P$ be a point of $K$.

If $K$ is a proper subset of $M$, and $Q$ is any point of $M-K$, any subcontinuum $N$ of $M$ which is irreducible between $P$ and $Q$ contains no points of $K$, except $P$, because $N-P-Q$ can contain no points having property 5 , and therefore none having property 3 , by Theorems 14 and 9 . Therefore $M-(N-P)$ contains $K$, and $P$ fails to have property 3 , which is contrary to hypothesis.

If $K$ is identical with $M$, any subcontinuum $N$ of $M$ which is irreducible between any two points $P$ and $Q$ of $M$, consists entirely of points of $K$. But no point of $N-P-Q$ can have property 5 , and therefore none can have property 3. Therefore no point of $N-P-Q$ is a point of $K$, which is contrary to hypothesis. Therefore Theorem 19 is true.

COROLLARY 19a. The set of points of a bounded continuum which have property 7 , is totally disconnected. ${ }^{*}$

Corollary 19b. The set of points of a bounded continuum which have both properties 5 and 9 is totally disconnected.

COROLlary 19c. The set of end points of a continuous curve is totally disconnected. $\dagger$

TheOrem 20. A bounded continuum which is irreducible between two of its points, cannot contain more than two points with property 5.

TheOREM 21. The set of points of a bounded continuum which have property 5 , contains no continuum.

COROLlary 21a. The set of points of a bounded continuum which have property 6 , contains no continuum.

However, the set of points of a bounded continuum which have property 9 may contain a continuum, as was shown in example 11. Similarly with the set of points having property 8 , and with the set having property 4 .

THEOREM 22. If $M$ is a bounded continuum, and $K$ is any subset of the set of points of $M$ which have property 5 , then $M-K$ is strongly connected.

* Menger, loc. cit., p. 283, Theorem V.

† Whyburn, loc. cit., Theorem 21. See footnote on p. 391. We have shown in Part 2 of this paper that an end point in Whyburn's sense is equivalent to an end point in Menger's sense, in the case of a continuous curve.

$\ddagger$ In Whyburn's Theorem 31 the second paragraph should be omitted, as his "end point" has property 5 , and therefore $P$ cannot be any point other than $A$ or $B$. 
If this is not true, then $M-K$ contains two points $P$ and $Q$ such that every subcontinuum of $M$ containing $P$ and $Q$ contains a point of $K$. In particular, any subcontinuum $N$ of $M$ irreducible between $P$ and $Q$ contains a point of $K$. But no point of $N-P-Q$ can have property 5 , and therefore no point $N-P-Q$ is a point of $K$, which is a contradiction.

Corollary 22a. If $M$ is a continuous curve, and $K$ is any subset of the end points of $M$, then $M-K$ is strongly connected.*

By example 11, we see that Theorem 22 is not true if property 5 is replaced by any of the properties 4,8 , or 9 . In fact, in that case $M-K$ may be disconnected. The theorem remains true, of course, if property 5 is replaced by any of the properties 3, 6, or 7 .

THEOREM 23. A necessary and sufficient condition that a bounded continuum $M$ be an acyclic continuous curve, is that every non-cut point have property 5.

The necessity of the condition follows from the fact that every non-cut point of an acyclic continuous curve is an end point, $\dagger$ and therefore has property 5 .

The condition is sufficient, because if every non-cut point of a bounded continuum $M$ has property 5 , the set of non-cut points of $M$ is identical with the set of points of $M$ which have property 5 . If $K$ is any subcontinuum of $M$, then $K$ contains a subcontinuum $N$ which is irreducible between two points $X$ and $Y$, and therefore no point of $N-X-Y$ has property 5 . In other words, every point of $N-X-Y$ is a cut point of $M$. But if every subcontinuum $K$ of $M$ contains uncountably many cut points of $M$, then $M$ is an acyclic continuous curve. $\ddagger$

The sufficiency of the condition can also be established by Theorem 22 and Whyburn's Theorem 32 .

Note that the condition remains necessary and sufficient if we replace property 5 by any of the stronger conditions 3,6 , or 7 . In case property 5 is replaced by property 4 or property 8 the condition is necessary, but not sufficient, as Example 11 shows. If property 5 is replaced by property 9, the condition is neither necessary nor sufficient.

Theorem 24. If a point $P$ of a bounded continuum $M$ has property 7 , then $P$ is a limit point of cut points of $M$.

W. L. Ayres, loc. cit., Theorem 6, p. 401.

† Wilder, loc. cit., Theorem 7, p. 358.

$\ddagger$ R. L. Moore, Concerning the cut-points of continuous curves, etc., Proceedings of the National Academy of Sciences, vol. 9 (1923), pp. 101-106. See Theorem E, p. 103. 
Given any positive number $\epsilon$, there is a cut point of $M$ whose distance from $P$ is less than $\epsilon$ and therefore $P$ is a limit point of cut points of $M$. Since a point having any of the properties 3-9 is a non-cut point of $M$, a point having property 7 is a non-cut point of $M$ which is a limit point of cut points. However, the converse is not true, as a point $P$ may be a non-cut point and a limit point of cut points, and still not have any of the properties 3-9, even if $M$ is a continuous curve. An example which shows this, is a continuous curve $M$ consisting of the continuum of example 2, plus the straight line intervals from $(0,0)$ to $(0,1)$, from $(0,1)$ to $(1,1)$, and from $(1,1)$ to $(1,0)$, where $P$ is the point $(0,0)$.

The above theorem is not true if we replace property 7 by any or all of the properties $3-6,8-9$, as is shown by example 10 , where $P$ has all 3-6, $8-9$, but is not a limit point of cut points of $M$.

Corollary 24a. If $P$ is an end point of a continuous curve $M$, then $P$ is a limit point of cut points of $M$.

We have already proved Corollary 24a incidentally in the proof of Theorem 1, where we used this fact to establish that if $P$ has property 1 , it has property 7.

Theorem 25. If a point $P$ of a bounded continuum $M$ has property 7 , then $M$ is connected im kleinen at $P$.

Given any positive number $\epsilon$, there is a domain $D$ containing $P$ of diameter less than $\epsilon$, and such that the set of points of $M$ in $D$ plus its boundary is a continuum $N$. If $\delta$ is any positive number which is less than the distance from $P$ to any point of the exterior of $D$, then any two points of $M$ at a distance less than $\delta$ from $P$ are points of $N$, and since $N$ is of diameter less than $\epsilon$, it follows that $M$ is connected im kleinen at $P$.

THEOREM 26. If a point $P$ of a bounded continuum $M$ has property 5 and property 9, then $M$ is connected im kleinen at $P$.

In the proof of Theorem 11, we showed that if $P$ has properties 5 and 9 , and if $N$ is any subcontinuum of $M$ containing $P$, then $P$ is not a limit point of the set $M-N$. Given any positive number $\epsilon$, let $N$ be a subcontinuum of $M$ of diameter less than $\epsilon$ and containing $P$, and let $\delta$ be less than the distance from $P$ to any point of $M-N$. Then as in the proof of Theorem 25, it follows that $M$ is connected im kleinen at $P$.

These two theorems might be combined into the single theorem that $M$ is connected im kleinen at $P$ if $M$ has properties 3-7, or 3-6, 8, and 9. Example 11 shows that $M$ is not necessarily connected im kleinen at $P$, if $P$ has proper- 
ties 4, 8 and 9, and example 8 shows that $P$ may have properties $3-6$, and 8 , and still $M$ need not be connected im kleinen at $P$. Therefore the hypothesis of neither of the above theorems can be weakened.

Whyburn* has shown that if a point $P$ of a bounded continuum $M$ has property 6 and is accessible from some point of a domain complementary to $M$, then $M$ is connected im kleinen at $P$. This naturally raises the question as to the conditions under which a point $P$ having certain of the given properties is accessible from a domain complementary to $M$.

If $M$ is a continuous curve, every point of $M$ (whether it be an end point or not) is accessible from every complementary domain on whose boundary the point lies. However, a point may be an end point of a continuous curve and also have property 8 , and still not be on the boundary of any complementary domain. An example which shows this, is the continuous curve $M$ consisting of the straight line interval from $(0,0)$ to $(1,0)$, and the set of circles $x^{2}+y^{2}=1 / n^{2}$, for $n=1,2,3, \cdots$. The point $P=(0,0)$ has properties $1-8$, but is not on the boundary of any complementary domain.

However, if an end point of a continuous curve has property 9, it is a boundary point of a complementary domain, by Theorem 4 .

If $M$ is a bounded continuum (not necessarily a continuous curve), a point $P$ may have all the properties 3-9, and still not be a boundary point of a complementary domain. An example which shows this, is the continuum consisting of the point $(0,0)$, the set of circles $x^{2}+y^{2}=1 / 4^{n}$, and the two sets of curves whose equations in polar coördinates are $r=(3+\theta /(\theta+1)) / 2^{n+2}$, for $\theta \geqq 0$, and $r=(3-\theta /(\theta+1)) / 2^{n+2}$, for $\theta \geqq 0$, and for $n=0,1,2, \cdots$. The point $P=(0,0)$ has properties $3-9$, but is not on the boundary of any domain complementary to $M$. Therefore, in the two following theorems, it is necessary to assume that $P$ is on the boundary of a complementary domain.

Theorem 27. If a point $P$ of a bounded continuum $M$ has property 7 and is on the boundary of a domain $D$ complementary to $M$, then $P$ is accessible from $D$.

Since $P$ has property 7 , if $C$ is any circle about $P$ as center, there exists a domain $E$ interior to $C$, containing $P$, whose exterior contains points of $D$, and whose boundary $F$ has just one point $X$ in common with $M$. The domain $E$ can also be selected so that $F-X$ is connected. $\dagger$ The domain $E$ contains

* Loc. cit., Theorem 30, p. 395.

$\dagger$ R. L. Moore, Concerning the sum of a countable number of mutually exchusive continua in the plane, Fundamenta Mathematicae, vol. 6 (1924), p. 191, Theorem 3. 
points of $D$, and since the exterior of $E$ also contains points of $D$, it follows that all points of $F-X$ are points of $D$. If $C_{1}$ is any circle about $P$ as center, and interior to $E$, any two points $P_{1}$ and $P_{2}$ of $D$ interior to $C_{1}$ can be joined by a connected subset of $D$ interior to $C$,-the connected subset consisting of $F-X$ plus the points in $E$ of any arc joining $P_{1}$ to $P_{2}$ in $D$. Hence $D$ is "connected near $P$ " and hence* $P$ is accessibli from $D$.

Theorem 28. If a point $P$ of a bounded continuum $M$ has property 5 and property 9 , and is on the boundary of a domain $D$ complementary to $M$, then $P$ is accessible from $D$.

If $B$ is the boundary of $D$, then $P$ has properties $3-6,8-9$ in the continuum $B$, by Theorem 18. Therefore $B-P$ is connected and $B$ contains no continuum of condensation containing $P$, and therefore $D$ is connected near $P$, and $P$ is accessible from $D$.†

Again, we might combine the above two theorems into the single theorem that $P$ is accessible from a complementary domain $D$, if $P$ is on the boundary of $D$, and has either properties 3-7, or properties 3-6, 8-9. Examples 8 and 11 show that neither hypothesis can be weakened and the theorem remain true, as these examples show that a point may have properties 4,8 , and 9 or properties $3-6$, and 8 , and be on the boundary of a complementary domain of $M$, and still not be accessible from that domain.

In conclusion, I wish to express my sincere appreciation to Professor R. L. Moore for his inspiring assistance in the preparation of all the papers written during my year as National Research Fellow.

* R. G. Lubben, Concerning connectedness near a point set.

† R. G. Lubben, loc. cit.

The University or Texas, Austin, Texas 